\title{
Evaluation of Knee Kinematics and Moments during Active Deep Flexion Activity after Oxford Mobile-Bearing Medial UKA-A Two-Year Follow-Up Study
}

\author{
Li Yue, MD ${ }^{1,20}$ Mario Lamontagne, $\mathrm{PhD}^{2} \quad$ Zhao Xiong, $\mathrm{PhD}^{2} \quad$ Zhu Zhengfei, $\mathrm{PhD}^{3}$ Tian Run, $\mathrm{MD}^{1}$ \\ Li Zhe, MD ${ }^{1}$ Kong Ning, MD ${ }^{1}$ Wang Chunsheng, $M^{1} \quad$ Yang Pei, MD ${ }^{1}$ Wang Kunzheng, MD ${ }^{1}$
}

\footnotetext{
${ }^{1}$ Department of Orthopaedics, The Second Affiliated Hospital of Xi'an Jiaotong University, Shaanxi Province, P.R. China

${ }^{2}$ School of Human Kinetics, University of Ottawa, Ottawa Ontario, Canada

${ }^{3}$ State Key Laboratory for Manufacturing Systems Engineering, School of Mechanical Engineering, Xi'an Jiaotong University, Xi'an, China
}

J Knee Surg 2022;35:896-903.

\begin{abstract}
Address for correspondence Yang Pei, MD, Department of Orthopaedics, The Second Affiliated Hospital of Xi'an jiaotong University, No. 157 Xiwu Road, 710004, Xi'an, Shaanxi Province, P.R. China (e-mail: yangpei@vip.163.com).
\end{abstract}

Few studies have assessed knee range of motion (ROM) and moments of patients with mobilebearing unicompartmental knee arthroplasty (MB UKA) during active deep flexion activities. We analyze knee kinematic and kinetic parameters during postoperative squatting-standing activity, aiming to evaluate the efficacy of MB UKA and postoperative rehabilitation progress. This was a clinical cohort study. We followed up with 37 patients diagnosed with medial knee osteoarthritis $(\mathrm{OA})$ with primary UKA. After screening 31 patients were recruited to take gait tests. Squatting-standing activities were performed under the test of 10-camera motion analysis system and force plates preoperatively at different stages after UKA $(12,18$, and 24 months). The average duration of follow-up was 24.4 months (from 22.8 to 26.7 months). Hipknee-ankle angle improved significantly compared with pre-UKA as well as scores of American Knee Society Score, numeric rating scale, ORS, and Western Ontario and McMasters. University Osteoarthritis Index. About 83.6\% (31/37) of follow-up patients completed squatting-standing activity independently. At 1-year follow-up, peak varus angle (20.6 \pm 2.8 degrees), internal rotation angle (13.6 \pm 1.8 degrees), extensor moment $\left(1.44 \pm 0.04 \mathrm{~N}^{*} \mathrm{~m} / \mathrm{kg}\right)$, and internal rotator moment $\left(0.02 \pm 0.005 \mathrm{~N}^{*} \mathrm{~m} / \mathrm{kg}\right)$ of UKA knees were inferior to contralateral knees. Peak adductor moment $\left(0.76 \pm 0.05 \mathrm{~N}^{*} \mathrm{~m} / \mathrm{kg}\right)$ was superior to contralateral knees. At 2-year follow-up, peak flexion angle $\left(125.0 \pm 2.8\right.$ degrees) showed a growing trend meanwhile extensor $\left(1.70 \pm 0.03 \mathrm{~N}^{*} \mathrm{~m} / \mathrm{kg}\right)$ and adductor $(0.68 \pm 0.06 \mathrm{~N} * \mathrm{~m} / \mathrm{kg})$ moment closely resembled those of the contralateral knee. MB UKA could alleviate the affected knee mainly in flexion-extension ROM and moment meanwhile did not affect the biomechanical indicators of healthy limbs. OA knees in the early postoperative period showed decreased extensor moment and increased adductor moment during active deep flexion activity. Better ROM and relatively more natural extensor and adductor moment of UKA knee with rehabilitation time increasing may predict ideal rehabilitation outcome in the medium or longer term. received

September 17, 2018

accepted after revision

October 5, 2020

published online

January 5, 2021 (c) 2021. Thieme. All rights reserved.

Thieme Medical Publishers, Inc.,

333 Seventh Avenue, 18th Floor,

New York, NY 10001, USA
DOI https://doi.org/

10.1055/s-0040-1721033. ISSN 1538-8506. 
Many countries are facing the trend of an aging population, and the incidence of degenerative knee disease is increasing year by year. Osteoarthritis $(\mathrm{OA})$ has been considered one of the most frequent causes of pain, loss of function, and disability in adults. ${ }^{1}$ This most common disease of the knee joint ${ }^{2}$ is mostly located in the medial compartment at the early stage. ${ }^{3}$ Arthroplasty is a thorough solution for patients who have received ineffective conservative treatment. ${ }^{4}$ Unicompartmental knee arthroplasty (UKA) has become an effective and feasible treatment for early knee OA, especially for medial compartment OA. ${ }^{5-9}$ Previous literatures have reported satisfactory functional outcomes after UKA. ${ }^{10-16}$ One clinical research ${ }^{17}$ demonstrated UKA results in a more physiological gait performance compared with total knee arthroplasty (TKA), and a higher top walking speed. Mobile-bearing (MB) UKA is considered to offer better restoration of the natural kinematics of the knee and reduction of contact stress and wear. ${ }^{18-20}$ Furthermore, it has been suggested that MB knees may be superior to fixed-bearing designs in their restoration of native tibiofemoral biomechanics, thus permitting more natural joint mechanics. ${ }^{21-23}$

Gait analysis has been widely applied in the field of knee joint surgery. Kinetic and kinematic gait variables have not only been associated with OA progression ${ }^{24}$ but also with postoperative functional outcome. ${ }^{25}$ Unlike TKA, Wiik et al ${ }^{26}$ revealed that UKA enables near normal gait at higher speeds, and that walking speed and stride length in UKA group seemed more similar to normal controls. Another study ${ }^{27}$ showed that although some abnormalities persisted in muscle activity around the knee, a good restoration of gait was achieved by most unicompartmental knee patients independently of the UKA design. Multiple UKA gait scene such as stair ascent, ${ }^{28}$ downhill walking, ${ }^{29}$ and on a level and sloped grounds ${ }^{30}$ have been studied to demonstrate the biomechanical characteristics of UKA.

However, little has been known about knee range of motion (ROM) and moments in six degree of freedom of patients with MB UKA during active deep flexion activities. Our present work described the knee ROM and moments of medial MB UKA knees at high flexion state in three-dimensional planes to find out whether there existed interlimb differences for knee kinetics and kinematics. By analyzing knee kinematic and kinetic parameters during postoperative squatting-standing activity, we aimed to evaluate the efficacy of MB UKA and postoperative rehabilitation progress.

\section{Materials and Methods}

\section{Patient Selection}

Thirty-seven consecutive unilateral UKAs between August 2016 and December 2018 in 34 patients diagnosed with medial knee OA using Oxford (Biomet UK Ltd, Swindon, UK) MB UKA implant at the orthopaedic department of the Second Affiliated Hospital of Xi'an Jiaotong University. All the UKAs were performed by the same experienced operation group. Both femoral and tibial implants were cemented.

Criteria for selecting the subjects were as follows:
The inclusion criteria are as follows: (1) UKA patients' age: from 60 to 80 years old; (2) knee OA staged as grade 1 or 2 based on the Kellgren \& Lawrence Radiographic Grading System(radiographic evaluation was performed by an independent experienced imaging specialist); (3) diagnosed with medial knee OA with primary UKA; (4) informed of the purpose, process, and cautions of this clinical study, and signed informed consent. The exclusion criteria are as follows: (1) body mass index greater than $26 \mathrm{~kg} / \mathrm{m}^{2}$; (2) previous knee or other lower limb joint surgery, lower extremity trauma, tumor, rheumatoid arthritis, and vascular or neurological diseases; (3) uncontrolled hypertension or diabetes; (4) activities of daily living (ADL) dysfunction, constant need for walking aids; (6) coexisting or progression OA in the contralateral knee; (7) complications such as infection, deep vein thrombosis, dislocation, prosthesis aseptic loosening after surgery.

After excluding nonstandard body mass index (3 cases), lack of muscle strength (1 cases), and progression in contralateral arthritis (2 cases), 31 cases out of 37 consecutive follow-up patients after UKA were selected as gait analysis group. All of the subjects could complete the squattingstanding activity independently, and they filled out the questionnaires completely and correctly, thus no patient or questionnaire had to be taken out of the study.

\section{Clinical Assessment}

All patients received the same standardized perioperative management strategy and postoperative rehabilitation program. During the recovery period, clinical assessments were performed to exclude infection, deep vein thrombosis, dislocation, prosthesis aseptic loosening after surgery and subjectively evaluate function and alignment. The following four clinical scores were assessed: American Knee Society Score (AKSS); Oxford Knee Score (OKS); Western Ontario and McMaster Universities Osteoarthritis Index (WOMAC); numeric rating scale (NRS).

\section{Study Protocol}

In the gait laboratory, we designed a new mode of action to examine kinetics and kinematics of each patient before and after (with an average of 7.4 months) implantation of MB UKA was used. We adopted the systematic default whole body labeling method, the so-called plug-in gait marker set (-Fig. 1). After the survey and body surface measurement, the recruited UKA patients completed the squatting-standing activity independently at four specified time points (preoperatively, 12, 18, and 24 months after UKA). Three-dimensional kinematic data of the trunk, pelvis, and lower extremity were recorded using a Vicon (Vicon Motion Systems Ltd., Centennial, CO) T40 10-camera motion capture system at $100 \mathrm{~Hz}$. Ground reaction force data were collected using two AMTI (Advanced Medical Technology Inc., Watertown, MA; OR6-7-2000) force platforms with a sampling frequency of $1000 \mathrm{~Hz}$. Every subject's initial state was upright with their feet pointing forward, feet and shoulders the same width, and their hands naturally falling apart on both sides of the body. We took the time point when barycenter began to drop as the beginning point of the 


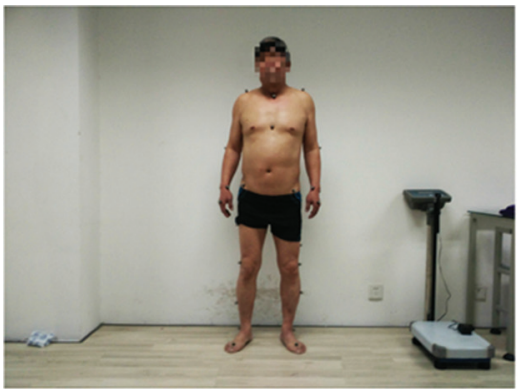

Front side

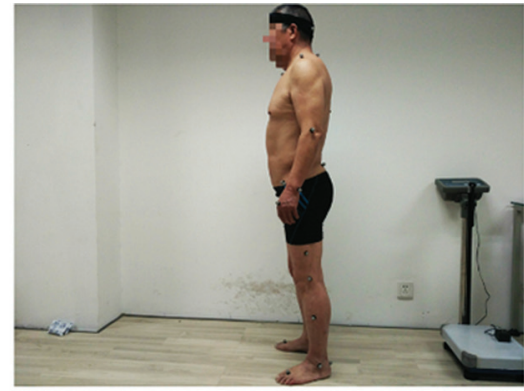

Lateral side

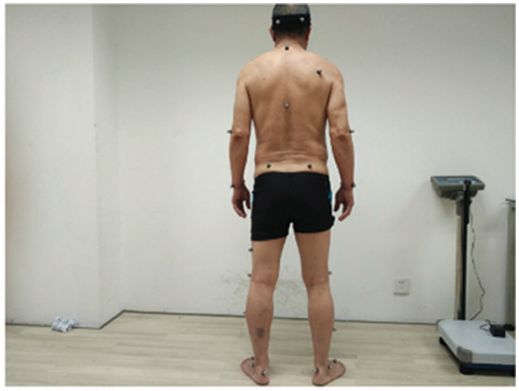

Back side

Fig. 1 Whole body labeling of unicompartmental knee arthroplasty patient by plug-in gait marker set.

squatting-standing cycle. After one cycle, the barycenter rose

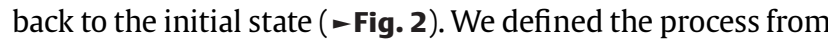
the initial state to back to the initial state as a squattingstanding cycle. Every subject was required to reach the maximum knee flexion angle according to respective ability. All the participants repeated the activity three times to obtain sufficient and reliable data. This study protocol was approved by the ethics committee of the Second Affiliated Hospital of Xi'an Jiaotong University.

\section{Post-processing and Statistical Analysis}

Pre- and postoperative clinical scores and gait parameters were compared with gait analysis improvements in the operated leg. Joint angles of the lower limbs were defined using Cardan angles. ${ }^{31}$ The joint angles exhibited during squattingstanding activity were adjusted to the joint angles displayed during natural standing. To generate the internal knee moments occurring during the activity, inverse dynamics procedures were performed using author-developed programs written in MATLAB 7.0 (MathWorks, Inc., Natick, MA). A fourth-order, low-pass Butterworth filter (cutoff frequency $=100 \mathrm{~Hz}$ ) was applied to the good reaction force and moment signals. Joint moments were aligned with segment axes as defined by International Society of Biomechanics recommendations ${ }^{32}$ and were scaled by body mass and leg length. The paired $t$-tests tests were two-sided to test interlimb differences within the pre- and postoperative UKA group, and a $p$-value of 0.05 was considered significant. Pearson's correlations were performed to investigate the relationship between peak flexion angle, peak extensor moment, peak adductor moments and postoperative time, and the $p$-values need to be corrected by Bonferroni. Statistical analysis was performed using SPSS version 23.0 for Windows (SPSS Inc., Chicago, IL).

\section{Results}

\section{Baseline Information of All Subjects}

All patients enrolled in the cohort received paper questionnaires. According to pre- and postoperative questionnaire survey of admitted patients, patient demographics are reported in -Table 1 .

\section{Clinical Scores and Related Assessments}

Compared with preoperative data, significant improvements were found in clinical scores and related assessments, as shown in - Table 2 . Pain reduced from 6.6 to $1.7(p<0.01)$ on

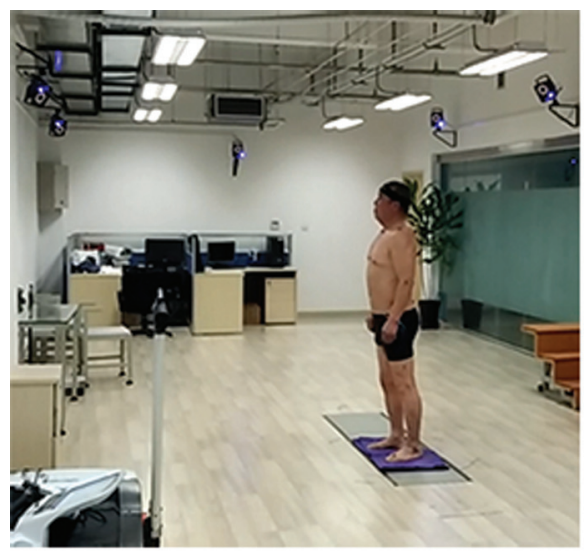

Standing phase

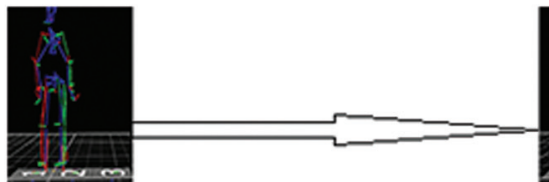

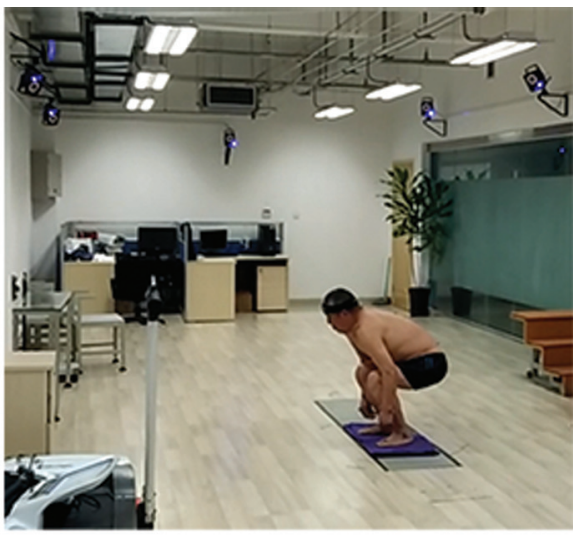

Squatting phase

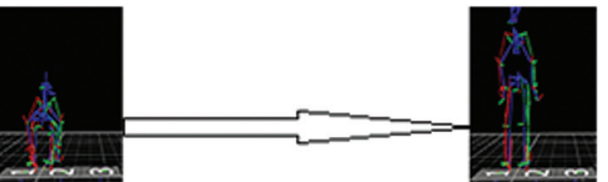

Fig. 2 Two typical phase of standing-squatting activity and schematic diagram of a complete squatting-standing cycle. 
Table 1 Demographics of participants

\begin{tabular}{|c|c|c|c|c|c|c|c|c|c|}
\hline $\begin{array}{l}\text { Number } \\
\text { of UKA } \\
\text { participants }\end{array}$ & $\begin{array}{l}\text { Gender } \\
\text { (male:female) }\end{array}$ & $\begin{array}{l}\text { Affected } \\
\text { limbs } \\
\text { (left:right) }\end{array}$ & Age (y) & Height(m) & $\begin{array}{l}\text { Weight } \\
\text { (kg) }\end{array}$ & $\begin{array}{l}\text { BMI } \\
\left(\mathrm{kg} / \mathrm{m}^{2}\right)\end{array}$ & $\begin{array}{l}\text { Leg } \\
\text { length } \\
(\mathrm{mm})\end{array}$ & $\begin{array}{l}\text { Knee } \\
\text { breadth } \\
(\mathrm{mm})\end{array}$ & $\begin{array}{l}\text { Total } \\
\text { postoperative } \\
\text { follow-up time } \\
\text { (mo) }\end{array}$ \\
\hline \multirow[t]{3}{*}{31} & $13: 18$ & $11: 20$ & $64.2 \pm 3.3$ & $1.63 \pm 0.11$ & $66.4 \pm 10.1$ & $23.7 \pm 1.4$ & $\begin{array}{l}\text { Healthy side } \\
784 \pm 29\end{array}$ & $\begin{array}{l}\text { healthy side1 } \\
05 \pm 4\end{array}$ & $24.4 \pm 1.3$ \\
\hline & & & & & & & $\begin{array}{l}\text { Affected side } \\
778 \pm 32\end{array}$ & $\begin{array}{l}\text { Affected side } \\
104 \pm 5\end{array}$ & \\
\hline & & & $(59-70)$ & & & $(22.0-25.7)$ & $(p=0.07)$ & $(p=0.68)$ & $(22.5-26)$ \\
\hline
\end{tabular}

Abbreviations: BMI, body mass index; UKA, unicompartmental knee arthroplasty.

Note: The values are indicated as means \pm standard deviation.

Table 2 Pre- and postoperative clinical assessments

\begin{tabular}{|l|l|l|l|}
\hline \multirow{2}{*}{$=31$} & Preoperative & 12 Mo follow-up & -Values \\
\cline { 2 - 4 } & (mean \pm SD) & (mean \pm SD) & \\
\hline NRS & $6.6 \pm 1.7$ & $1.7 \pm 1.4$ & $<0.01$ \\
\hline AKSS function & $63 \pm 19$ & $88 \pm 14$ & $<0.01$ \\
\hline AKSS motion & $47 \pm 13$ & $86 \pm 16$ & $<0.01$ \\
\hline WOMAC & $53 \pm 6$ & $22 \pm 3$ & $<0.01$ \\
\hline OKS & $38 \pm 5$ & $19 \pm 3$ & $<0.01$ \\
\hline $\begin{array}{l}\text { HKA angle } \\
\text { of affected } \\
\text { limb (degrees) }\end{array}$ & $6.9 \pm 2.7$ & $2.2 \pm 2.0$ & $<0.01$ \\
\hline
\end{tabular}

Abbreviations: AKSS, American Knee Society Score; NRS, numeric rating scale; OKS, Oxford Knee Score; SD, standard deviation; WOMAC, Western Ontario and McMaster Universities Osteoarthritis Index. Note: The values are indicated as means \pm standard deviation.

the NRS. AKSS increased significantly from 63 to $88(p<0.01)$ in function and from 47 to 86 in motion. WOMAC and NRS decreased from 28.9 to $17.8(p<0.01)$ postoperatively. As for measurement of lower limb alignment by imaging follow-up, hip-knee-ankle angle of affected limb restored from 6.9 to 2.2 degrees $(p<0.01)$.

\section{Kinematics and Kinetics of UKA and Healthy Limbs}

No significant differences ( $p=0.23$ ) were found between pre$(3.9 \pm 0.36 \mathrm{~s})$ and postoperative $(4.0 \pm 0.28 \mathrm{~s})$ squattingstanding cycle. The peak flexion angle of the affected knee rose from 108.5 degrees before the operation to 113.6 degrees with a $p$-value $<0.001$. The peak varus angle of the affected knee shifted by 2.8 degrees from 23.4 degrees preoperatively to 20.6 degrees postoperatively $(p=0.001)$. No significant reduction in post-UKA affected limbs' peak flexion and varus angle was found compared with post-UKA healthy limbs $(p=0.42, p=0.50)$. Kinematics and kinetics parameters in one squatting-standing cycle presented a characteristic pattern, as can be seen from the - Fig. 3 . Judging from the graph line trend, the moment parameters were quite different compared with the angle parameters. The former showed the trend of different changing rhythm between affected and healthy lower limbs after UKA. As for
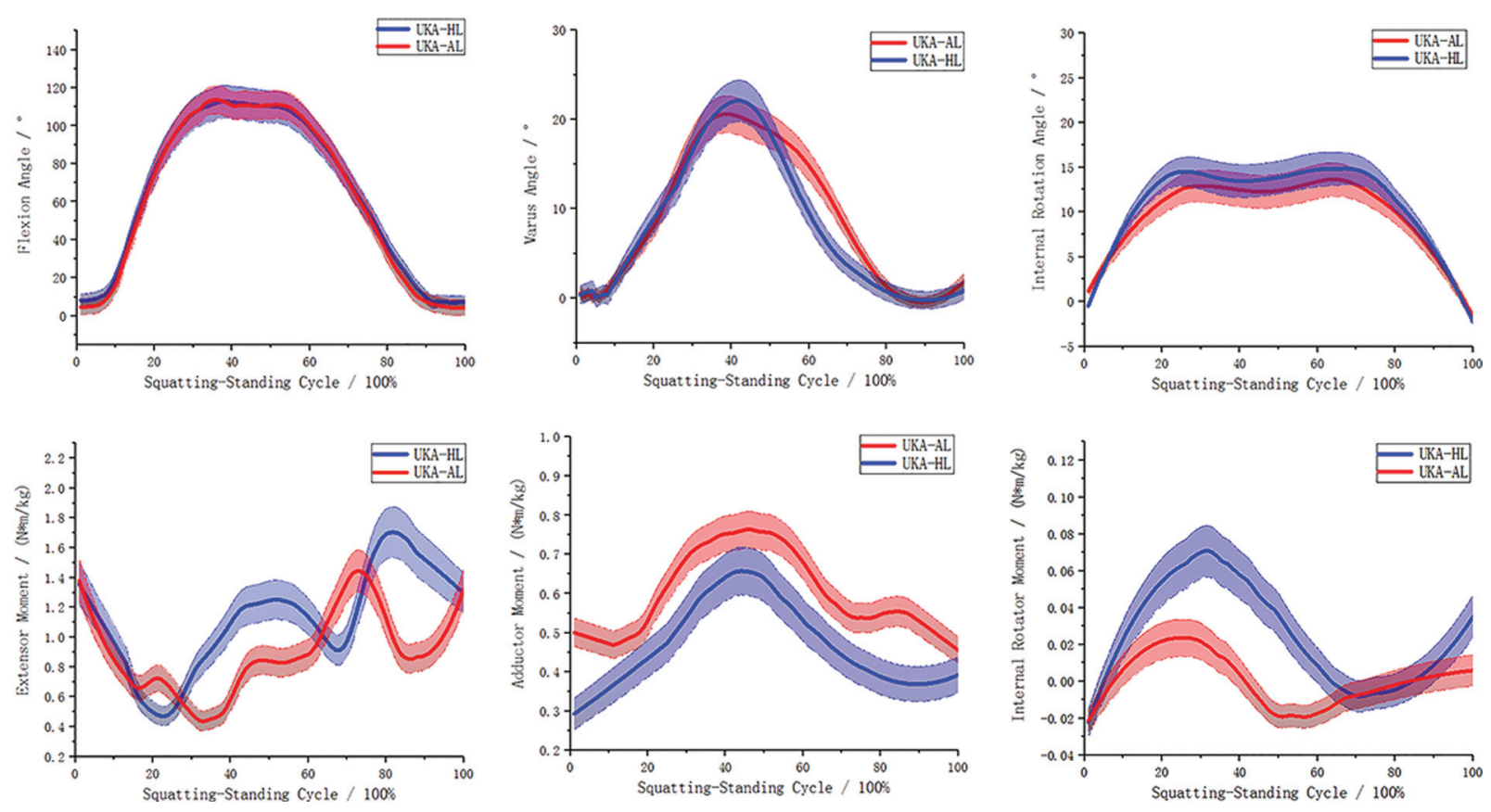

Fig. 3 Variation curves of knee motion angles (Graph A, B, and C) and moments (Graph D, E, and F) in three-dimensional planes during one squatting-standing cycle. Solid lines represent the average values and shaded regions the standard deviations. UKA-HL, unicompartmental knee arthroplasty patients' healthy limbs; UKA-AL, unicompartmental knee arthroplasty patients' affected limbs. 
Evaluation of Knee Kinematics and Moments during Active Deep Flexion Activity after Oxford Mobile-Bearing Medial

moment parameter analysis, extensor moments of post-UKA affected limbs decreased significantly compared with preUKA affected limbs $(p<0.01)$ and post-UKA healthy limbs $(p<0.01)$. On the contrary, adductor moments of post-UKA affected limbs increased significantly compared with the other two groups ( $p<0.01$, respectively). The main differences between affected limbs and healthy limbs before UKA were flexion-extension ROM and moment. Each individual self-comparison before and after the UKA operation showed that apart from the difference in abductor torque, there was no significant difference for healthy limbs in movement and torque in other directions ( $\mathbf{- T a b l e ~} \mathbf{3}$ ).

\section{Key Kinematics and Kinetics Parameters Change with Rehabilitation Progression}

The results of the correlational analysis were presented in - Fig. 4. With the postoperative recovery time $(12,18$, and 24 months) increasing, the knee peak flexion angle and extensor moment showed a gradual upward trend, and the peak adductor moment presented a downward trend. Positive correlations were found between peak flexion angle, extensor moment, adductor moment, and postoperative time $(r=0.625,0.854,-0.736 ; p=0.005,0.004,0.005 ; p$ values were corrected by Bonferroni).

\section{Discussion}

Although UKA as a minimally invasive surgery has become more and more popular in recent decades, ${ }^{7,33}$ the biomechanics of patients with UKAs have been poorly described and reported. We are accustomed to evaluating surgical outcomes through imaging and functional scores. A 12year Chinese follow-up ${ }^{34}$ showed UKA is a good option for the treatment of anteromedial OA of the knee. The mean OKS increased from 22.5 to 38.5 points, while the mean Knee Society Score (KSS) increased from 43.6 to 86.1 points. The mean visual analog scale pain score decreased from 7.9 to 1.5 points and the mean ROM increased from 112.5 to 125.2 degrees. However, postoperative radiographic evaluation is often not associated with functional scores. ${ }^{35}$ Postoperative radiographic changes in tibiofemoral alignment and joint space are only static observation result. Postoperative follow-up and functional scoring are influenced by subjective factors and do not directly explain UKA patients' recovery process. A successful UKA surgery means a good and stable state of function presented in patients' daily lives. Gait analysis has been used as a diagnostic instrument for measuring changes in the gait pattern before and after medial $M B$ UKA. Besides level walking, ramp walking, and upstairs, more complicated gait patterns close to daily life need to be studied and analyzed. When squatting normally, the knee flexion angle can be up to 130 to 135 degrees, and the knee load is five to eight times the body weight. Unlike western cultures, high flexion of the knee joint is very common in Asian life. For example, squatting devices for toilet require high flexion and heavy load. Therefore, it is meaningful to evaluate the squatting activity of patients after UKA to restore their quality of life better.

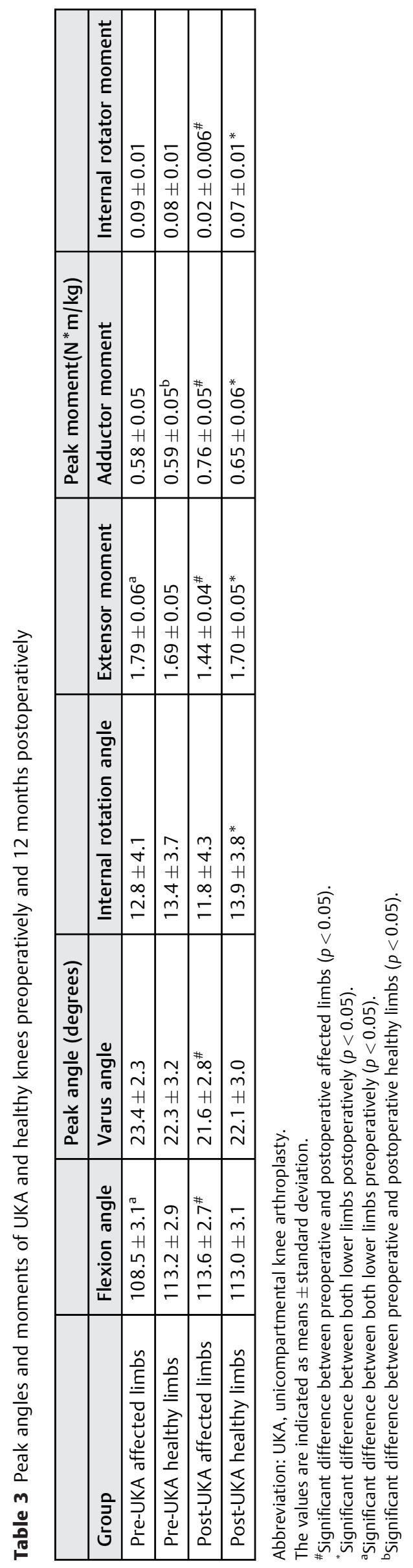



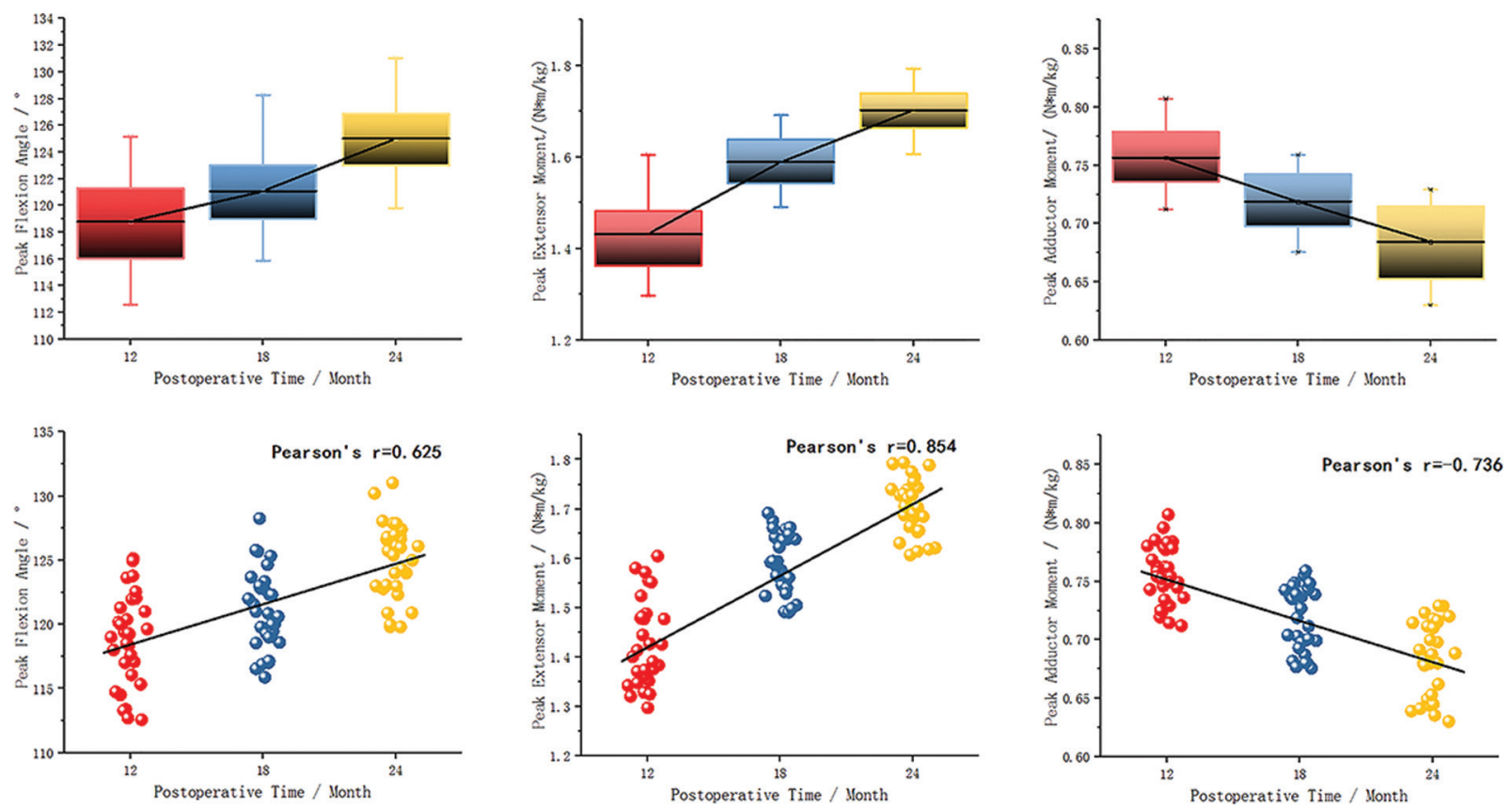

Fig. 4 Box plots (A-C) and scatter plots (D-F) display peak flexion angles, peak extensor moments, and peak adductor moments change over postoperative time. Regression lines and Pearson's correlation $r$ are noted in Graph D, E, and F.

The most important finding made in this study was that OA knees after medial MB UKA showed decreased extensor moment and increased adduction moment during active deep flexion activity. Our hypothesis was that self-protection mechanism of affected limbs and compensatory mechanism of healthy limbs were common in most UKA patients. Twelve months after surgery although the peak flexion angle, various clinical scores, and alignment evaluation significantly improved, there was still a big gap between lower extremities' moments. The two lower limbs of patients exhibited obvious asymmetry. With the progress of postoperative recovery, the situation obviously ameliorated. We recommended that knee exercises were performed step by step: Passive and active ROM-basic gait-ADL-senior functional activities. And the training program schedule was dependent on the functional outcome of each patient. We found continuous muscle exercise is essential in the early and mid-term after UKA surgery. As mentioned in the previous literature, the lack of medial compression leads to a tighter medial collateral ligament, and this drives the trend toward a stiffer joint by a decrease in frontal plane ROM. ${ }^{36}$ Interactively, restricting forward movement of the knees may minimize stress on the knees, and it is likely that forces are inappropriately transferred to the hips and low-back region. ${ }^{37}$ Therefore, it is also necessary to strengthen psoas and gluteal muscle exercise after UKA to ensure the stability of the knee.

Last but not the least, we have some thoughts about the overall design of this study. The majority of knee prostheses are TKA prostheses, including posterior-stabilized, cruciateretaining, medial-pivot, and hinged TKA prostheses. There's a big difference when it comes to the indications for UKA and TKA. From previous studies and experience, UKA patients have similar indications as high tibial osteotomy patients rather than TKA. ${ }^{38-40}$ Therefore, we think it may not be appropriate to compare the biomechanics indexes of patients after UKA and TKA. Because of less severe UKA indications, limbs after UKA surgery are expected to have relatively close performance as healthy limbs in gait. Because of the larger age distribution of patients undergoing surgery, it is not appropriate to compare their gait with healthy people of other age groups. Therefore, we finally adopted the research idea of comparing their own both lower extremities. Our study was limited by follow-up time. We were unable to track patients for 3 to 5 years and did not collect the data of long-term complications and survival rates. Further supplementary experiments were needed. Uncontrollable influences may play an important role in gait analysis studies. Substantial methodologic differences between studies may contribute to inconsistencies in reported findings for many gait outcomes. ${ }^{41}$ Because of the relatively big overall deviation, internal rotation angle and internal rotator moment were not fully analyzed and discussed. When considering the effects of implants, Oxford MB UKA implant still needs to be compared with other prosthesis types to prove its superiority in high flexion activity. For security reasons, railings or handrails need to be installed to provide support and prevent patients from falling hurt.

The clinical effect of UKA is an outcome of the prosthesis design in combination with the surgical technique and postoperative rehabilitation. Instrumented gait analysis helps to enable the surgeons to document time-distance parameters, joint kinematics, and kinetics. It is used not just for diagnostic purposes but also to monitor and evaluate the effects of surgical treatments. With the help of various comprehensive assessment tools, subjective and objective satisfactory results after UKA and being able to return to normal life without assistive devices and pain are our constant goals. 


\section{Conclusions}

MB UKA could alleviate the affected knee mainly in flexionextension ROM and moment meanwhile did not affect the biomechanical indicators of healthy limbs. OA knees in the early postoperative period showed decreased extensor moment and increased adductor moment during active deep flexion activity. Better ROM and relatively more natural extensor and adductor moment of UKA knee with rehabilitation time increasing may predict ideal rehabilitation outcome in the medium or longer term.

\section{Funding}

This study was supported by National Natural Science Foundation of China (General Program 81672173).

\section{Conflict of interest \\ None declared.}

\section{References}

1 Englund M, Turkiewicz A. [Osteoarthritis increasingly common public disease]. Lakartidningen 2014;111(21):930-931

2 Michael JW, Schlüter-Brust KU, Eysel P. The epidemiology, etiology, diagnosis, and treatment of osteoarthritis of the knee. Dtsch Arztebl Int 2010;107(09):152-162

3 Felson DT, Nevitt MC, Zhang Y, et al. High prevalence of lateral knee osteoarthritis in Beijing Chinese compared with Framingham Caucasian subjects. Arthritis Rheum 2002;46(05):1217-1222

4 Giwnewer U, Rubin G, Orbach H, Rozen N. [Treatment for osteoarthritis of the knee]. Harefuah 2016;155(07):403-406

5 Halawi MJ, Barsoum WK. Unicondylar knee arthroplasty: key concepts. J Clin Orthop Trauma 2017;8(01):11-13

6 Iacono F, Raspugli GF, Akkawi I, et al. Unicompartmental knee arthroplasty in patients over 75 years: a definitive solution? Arch Orthop Trauma Surg 2016;136(01):117-123

7 Murray DW, Parkinson RW. Usage of unicompartmental knee arthroplasty. Bone Joint J 2018;100-B(04):432-435

8 Amin AK, Patton JT, Cook RE, Gaston M, Brenkel IJ. Unicompartmental or total knee arthroplasty?: Results from a matched study Clin Orthop Relat Res 2006;451(451):101-106

9 Schindler OS, Scott WN, Scuderi GR. The practice of unicompartmental knee arthroplasty in the United Kingdom. J Orthop Surg (Hong Kong) 2010;18(03):312-319

10 Akizuki S, Mueller JK, Horiuchi H, Matsunaga D, Shibakawa A, Komistek RD. In vivo determination of kinematics for subjects having a Zimmer Unicompartmental High Flex Knee System. J Arthroplasty 2009;24(06):963-971

11 Banks SA, Fregly BJ, Boniforti F, Reinschmidt C, Romagnoli S. Comparing in vivo kinematics of unicondylar and bi-unicondylar knee replacements. Knee Surg Sports Traumatol Arthrosc 2005; 13(07):551-556

12 Chassin EP, Mikosz RP, Andriacchi TP, Rosenberg AG. Functional analysis of cemented medial unicompartmental knee arthroplasty. J Arthroplasty 1996;11(05):553-559

13 Fuchs S, Tibesku CO, Frisse D, Laass H, Rosenbaum D. Quality of life and gait after unicondylar knee prosthesis are inferior to age-matched control subjects. Am J Phys Med Rehabil 2003;82(06):441-446

14 Pandit H, Van Duren BH, Gallagher JA, et al. Combined anterior cruciate reconstruction and Oxford unicompartmental knee arthroplasty: in vivo kinematics. Knee 2008;15(02):101-106

15 Webster KE, Wittwer JE, Feller JA. Quantitative gait analysis after medial unicompartmental knee arthroplasty for osteoarthritis. J Arthroplasty 2003;18(06):751-759
16 Weinstein JN, Andriacchi TP, Galante J. Factors influencing walking and stairclimbing following unicompartmental knee arthroplasty. J Arthroplasty 1986;1(02):109-115

17 Jones GG, Kotti M, Wiik AV, et al. Gait comparison of unicompartmental and total knee arthroplasties with healthy controls. Bone Joint J 2016;98-B(10, Supple B):16-21

18 Ashraf T, Newman JH, Desai VV, Beard D, Nevelos JE. Polyethylene wear in a non-congruous unicompartmental knee replacement: a retrieval analysis. Knee 2004;11(03):177-181

19 Psychoyios V, Crawford RW, O'Connor JJ, Murray DW. Wear of congruent meniscal bearings in unicompartmental knee arthroplasty: a retrieval study of 16 specimens. J Bone Joint Surg $\mathrm{Br}$ 1998;80(06):976-982

20 Simpson DJ, Gray H, D'Lima D, Murray DW, Gill HS. The effect of bearing congruency, thickness and alignment on the stresses in unicompartmental knee replacements. Clin Biomech (Bristol, Avon) 2008;23(09):1148-1157

21 Smith TO, Hing CB, Davies L, Donell ST. Fixed versus mobile bearing unicompartmental knee replacement: a meta-analysis. Orthop Traumatol Surg Res 2009;95(08):599-605

22 Gleeson RE, Evans R, Ackroyd CE, Webb J, Newman JH. Fixed or mobile bearing unicompartmental knee replacement? A comparative cohort study. Knee 2004;11(05):379-384

23 Li MG, Yao F, Joss B, Ioppolo J, Nivbrant B, Wood D. Mobile vs. fixed bearing unicondylar knee arthroplasty: a randomized study on short term clinical outcomes and knee kinematics. Knee 2006;13 (05):365-370

24 Favre J, Jolles BM. Gait analysis of patients with knee osteoarthritis highlights a pathological mechanical pathway and provides a basis for therapeutic interventions. EFORT Open Rev 2017;1(10): 368-374

25 Rahman J, Tang Q Monda M, Miles J, McCarthy I. Gait assessment as a functional outcome measure in total knee arthroplasty: a cross-sectional study. BMC Musculoskelet Disord 2015;16:66

26 Wiik AV, Manning V, Strachan RK, Amis AA, Cobb JP. Unicompartmental knee arthroplasty enables near normal gait at higher speeds, unlike total knee arthroplasty. J Arthroplasty 2013;28 (09):176-178

27 Catani F, Benedetti MG, Bianchi L, Marchionni V, Giannini S, Leardini A. Muscle activity around the knee and gait performance in unicompartmental knee arthroplasty patients: a comparative study on fixed- and mobile-bearing designs. Knee Surg Sports Traumatol Arthrosc 2012;20(06):1042-1048

28 Fu YC, Simpson KJ, Brown C, Kinsey TL, Mahoney OM. Knee moments after unicompartmental knee arthroplasty during stair ascent. Clin Orthop Relat Res 2014;472(01):78-85

29 Wiik AV, Aqil A, Tankard S, Amis AA, Cobb JP. Downhill walking gait pattern discriminates between types of knee arthroplasty: improved physiological knee functionality in UKA versus TKA. Knee Surg Sports Traumatol Arthrosc 2015;23(06):1748-1755

30 Komnik I, Peters M, Funken J, David S, Weiss S, Potthast W. Nonsagittal knee joint kinematics and kinetics during gait on level and sloped grounds with unicompartmental and total knee arthroplasty patients. PLoS One 2016;11(12):e0168566. Doi: 10.1371/ journal.pone.0168566

31 Grood ES, Suntay WJ. A joint coordinate system for the clinical description of three-dimensional motions: application to the knee. J Biomech Eng 1983;105(02):136-144

$32 \mathrm{Wu}$ G, Siegler S, Allard PStandardization and Terminology Committee of the International Society of Biomechanics International Society of Biomechanics. et al. ISB recommendation on definitions of joint coordinate system of various joints for the reporting of human joint motion-part I: ankle, hip, and spine. J Biomech 2002; 35(04):543-548

33 Riddle DL, Jiranek WA, McGlynn FJ. Yearly incidence of unicompartmental knee arthroplasty in the United States. J Arthroplasty 2008;23(03):408-412 
34 Xue H, Tu Y, Ma T, Wen T, Yang T, Cai M. Up to twelve year followup of the Oxford phase three unicompartmental knee replacement in China: seven hundred and eight knees from an independent centre. Int Orthop 2017;41(08):1571-1577

$35 \mathrm{Kim} \mathrm{KT}$, Lee S, Kim TW, Lee JS, Boo KH. The influence of postoperative tibiofemoral alignment on the clinical results of unicompartmental knee arthroplasty. Knee Surg Relat Res 2012; 24(02):85-90

36 Heyse TJ, Tucker SM, Rajak Y, et al. Frontal plane stability following UKA in a biomechanical study. Arch Orthop Trauma Surg 2015;135(06):857-865

37 Fry AC, Smith JC, Schilling BK. Effect of knee position on hip and knee torques during the barbell squat. J Strength Cond Res 2003; 17(04):629-633
38 Jeon YS, Ahn $\mathrm{CH}$, Kim MK. Comparison of HTO with articular cartilage surgery and UKA in unicompartmental OA.J Orthop Surg (Hong Kong) 2017;25(01):2309499016684092

39 Cao Z, Mai X, Wang J, Feng E, Huang Y. Unicompartmental knee arthroplasty vs high tibial osteotomy for knee osteoarthritis: a systematic review and meta-analysis. J Arthroplasty 2018;33 (03):952-959

40 Lee YS, Kim HJ, Mok SJ, Lee OS. Similar outcome, but different surgical requirement in conversion total knee arthroplasty following high tibial osteotomy and unicompartmental knee arthroplasty: a meta-analysis. J Knee Surg 2019;32(07):686-700

41 McClelland JA, Webster KE, Feller JA. Gait analysis of patients following total knee replacement: a systematic review. Knee 2007;14(04):253-263 